\title{
Long-term tumor control of spinal dissemination of cerebellar glioblastoma multiforme by combined adjuvant bevacizumab antibody therapy: a case report
}

\author{
Thomas Linsenmann ${ }^{*}$, Camelia M Monoranu², Giles H Vince ${ }^{3}$, Thomas Westermaier ${ }^{1}$, Carsten Hagemann ${ }^{1}$, \\ Almuth F Kessler ${ }^{1}$, Ralf-Ingo Ernestus ${ }^{1}$ and Mario Löhr ${ }^{1}$
}

\begin{abstract}
Background: Glioblastoma multiforme located in the posterior fossa is extremely rare with a frequency up to 3.4\% Compared with glioblastoma of the hemispheres the prognosis of infratentorial glioblastoma seems to be slightly better. Absence of brainstem invasion and low expression rates of epidermal growth factor receptor are described as factors for long-time survival due to the higher radiosensitivity of these tumors.
\end{abstract}

Case presentation: In this case study, we report a German female patient with an exophytic glioblastoma multiforme arising from the cerebellar tonsil and a secondary spinal manifestation. Furthermore, the tumor showed no $\mathrm{O}$ (6)-Methylguanine-DNA methyltransferase promotor-hypermethylation and no isocitrate dehydrogenase 1 mutations. All these signs are accompanied by significantly shorter median overall survival. A long-term tumor control of the spinal metastases was achieved by a combined temozolomide/bevacizumab and irradiation therapy, as part of a standard care administered by the treating physician team.

Conclusion: To our knowledge this is the first published case of a combined cerebellar exophytic glioblastoma with a subsequent solid spinal manifestation. Furthermore this case demonstrates a benefit undergoing this special adjuvant therapy regime in terms of overall survival. Due to the limited overall prognosis of the disease, spinal manifestations of glioma are rarely clinically relevant. The results of our instructive case, however, with a positive effect on both life quality and survival warrant treating future patients in the frame of a prospective clinical study.

Keywords: Glioblastoma, Spinal dissemination, Bevacizumab, Temozolomide, Irradiation

\section{Background}

Glioblastoma mulitforme (GBM) is the most common primary tumor of the central nervous system (CNS) comprising approximately $50 \%$ of all primary intracranial tumors [1]. It is located most frequently in the cerebral hemispheres and the peak age of onset is the sixth or seventh decade. GBM located in the cerebellum is extremely rare with a frequency of $0 \%$ to $3.4 \%$ [2-5]. The standard treatment is surgical tumor excision to the extent feasible, followed by radiotherapy and temozolomide chemotherapy [6,7]. Compared to GBM of the hemispheres, the prognosis of infratentorial GBM

\footnotetext{
* Correspondence: Linsenmann_T@ukw.de

'Department of Neurosurgery, Julius-Maximilians-University, 97080 Wuerzburg, Germany

Full list of author information is available at the end of the article
}

seems to be slightly better. Absence of brainstem invasion and a low expression rate of epidermal growth factor receptor (EGFR) are described as factors for long-time survival due to the higher radiosensitivity of these tumors $[8,9]$.

Extracranial metastasis from GBM is rare, with a reported frequency of only $0.44 \%$. In the reported cases, metastases occurred in regional lymph nodes, lungs, pleura, and occasionally in the bone and liver. Spinal metastases are clinically rarely detected [10]. Spinal cord involvement can be due to a leptomeningeal or vertebral localization, whereas intraparenchymal metastasis of the myelon is extremely rare. Although involvement of the spinal cord has 


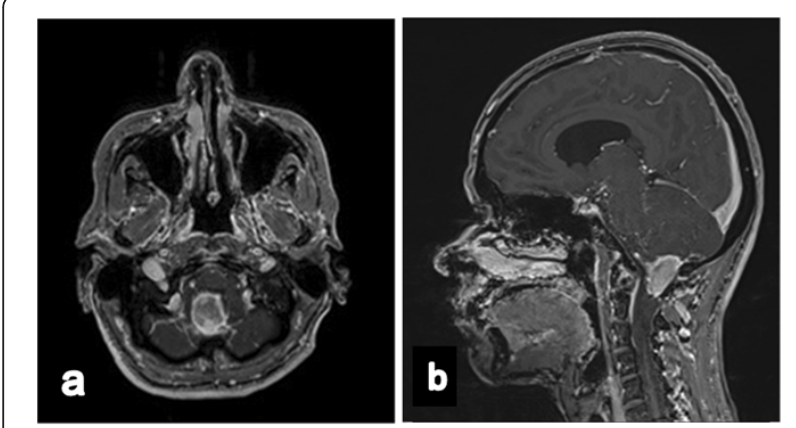

Figure 1 Preoperative (a) axial and (b) sagittal T1-weighted magnetic resonance imaging after gadopentetate dimeglumine application, demonstrating a well-defined enhancing mass located dorsal from the medulla oblongata with brainstem attachment.

been noted with increasing frequency in recent years, the literature provides 42 documented cases [11].

\section{Case presentation}

A 55-year-old German woman presented at our department with a short history of persistent headache, vertigo, nausea, and a generalized seizure. Magnetic resonance imaging (MRI) showed a well-defined tumor approximately $1.7 \times 3.7 \times 2.6 \mathrm{~cm}$ in size, located at the cranio-cervical junction with dorsal compression of the medulla oblongata, and in close relation to the right vertebral artery. The tumor was hyperintense on T2-weighted images and displayed an intense, nearly homogeneous gadolinium (Gd) enhancement. Due to its close contact to the dura, the initial tentative neuroradiological diagnosis was an infratentorial meningioma [Figure 1]. The tumor was completely removed via a median suboccipital craniotomy, opening of the foramen magnum, and resection of the dorsal arch of $\mathrm{C} 1$. Microsurgical complete excision of the tumor was achieved by the aid of electrophysiological monitoring (Electromyographies (EMG) of the lower cranial nerves, somatosensory evoked potentials (SEP) and motoric-evoked potentials of the medianus nerve (MEP)).

The histological examination on formalin fixed and paraffin embedded tissue confirmed the diagnosis of a glioblastoma multiforme WHO IV with a typical high cellular pleomorphism, microvascular proliferations, and necrotic areas [Figure 2] but without EGFR-expression. Immunhistochemistry also showed a missing MGMT O (6)-Methylguanine-DNA methyltransferase promotorhypermethylation (MGMT) and no isocitrate dehydrogenase 1 mutation (IDH1).

The postoperative course of the patient was uneventful. She underwent radiochemotherapy with an over-all dose of 54 Gy applied in fractions of 1,8 Gy twice a day over a period of 3 weeks and concomitant temozolomide chemotherapy $\left(75 \mathrm{mg} / \mathrm{m}^{2}\right.$ body surface). Adjuvant temozolomide chemotherapy according to the Stupp regimen (200 mg/m $\mathrm{m}^{2}$ body surface) [8] was administered 12 times. After 15 months without new tumor progression MRIs showed in the area of the right cerebellar tonsil a relapse of about $1.4 \times 1.9 \times 1.6 \mathrm{~cm}$ in size.

She underwent a second operation, but because the tumor had infiltrated the cranial nerves IX and X only a subtotal tumor resection could be performed.

The histological examination confirmed GBM with high mitotic rate and again negative MGMT methylation.

Two weeks later, the patient described lower limb pain, reduced sensitivity, and micturition disorders accompanied by progressive dysphagia. The spinal MRI revealed a small tumor located dorsal to the $4^{\text {th }}$ lumbar vertebral body and a large spinal tumor manifestation dorsal to L5 to $\mathrm{S} 1$ of approximately $4.0 \times 1.2 \mathrm{~cm}$ in the sagittal plane accompanied by multiple gadolinium - enhancing (gd) nodules throughout the whole spinal column [Figure 3]. Diagnosis of a meningeosis carcinomatosa was confirmed by the histological results of a spinal tap.

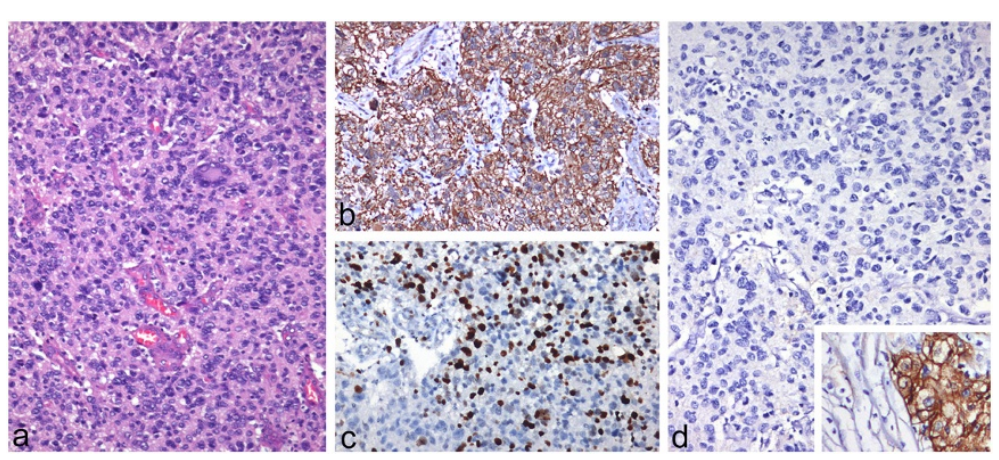

Figure 2 Histological examination on formalin fixed and paraffin embedded tissue. a) Glioblastoma with high cellular pleomorphism and microvascular proliferation ( $\mathrm{H \& E}, \mathrm{x100)}$; b) immunohistochemistry for glial fibrillary acidic protein shows strong expression in tumor cells (x100); c) high proliferation as determined by the antibody Ki67 ( $\times 100)$; d) lack of epidermal growth factor receptor in glioblastoma cells ( $x 100)$; in higher magnification (x200) colon carcinoma as positive control (inset). 


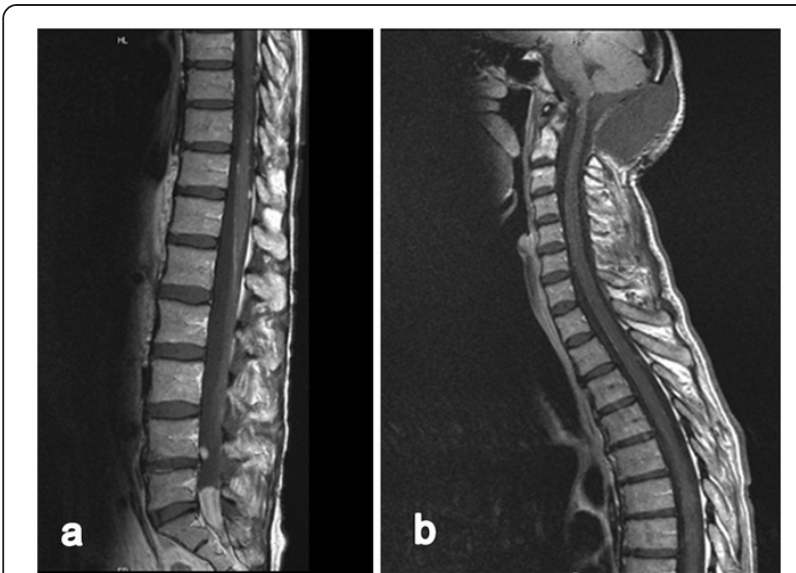

Figure 3 Sagittal T1-weighted magnetic resonance images enhanced with gadopentetate dimeglumine demonstrating spinal tumor located dorsal of lumbar vertebral body $L 4$ and $a$ large tumor manifestation dorsal L5 to S1 (a) of approximately $4.0 \times 1.2 \mathrm{~cm}$ in the sagittal view accompanied by multiple small enhancements in the range of the whole spinal cord $(a, b)$.

Radiotherapy was administered to the lumbar spinal mass with an over-all dose of 54 Gy administered in 18 sessions. The cerebellar relapse was treated likewise with 30 Gy in 5 sessions. Irradiation was combined with continuous temozolomide chemotherapy ("one week on, one week off", $120 \mathrm{mg} /$ day). After therapy the patient presented in an improved overall condition. She still described dysesthesia in the lower limbs but showed a normal function of the bladder and she was also able to walk without aid.

After radio-/chemotherapy the patient consented to off-label intravenous bevacizumab antibody-therapy with $15 \mathrm{mg} / \mathrm{kg}$ d1 q21d (according $800 \mathrm{mg}$ bevacizumab) combined with a continuous application of temozolomide $100 \mathrm{mg}$ per day over 25 bouts of therapy, administered by the treating physician team as part of a standard care in case of disseminated GBM.

This procedure is in consistent with the institutional ethics committee instructions according to the Federal Institute for Drugs and Medical Devices. The use is permitted within therapeutic flexibility of the physician in cases of life-threatening diseases without alternative therapeutic option and evidence for therapeutic success ( $\$ 35 \mathrm{bSGBV})$.

After 3 months of combined temozolomide/bevacizumab therapy the MRI scans revealed a decrease of cerebellar gd - enhancement and a stable clinical condition concerning the spinal manifestation such as dysesthesia of the lower limbs.

33 months after the first operation and 12 months after spinal irradiation the, MRI scans still showed stable disease without evidence of further tumor growth or new CNS manifestations [Figure 4] being aware the fact that it is difficult to discriminate the effects of the RT and the effects of the adjuvant bevacizumab/temozolomide therapy on the spinal cord. The patient presented in a reduced nutritional and general condition with dysphagia, vertigo combined with persistent weakness of the lower limbs, and atypical pneumonia mainly a result of affected caudal cranial nerves, which was not seen in the MRI yet. Wellknown side effects under therapy like progressive fatigue and nausea were also observed, but we also realised a good toleration of the therapy in haemograms.

The most recent MRI scan, 38 months after the first operation, revealed a progressive disease with extended meningeosis carcinomatosa around the brainstem and the cervical spinal cord. Because of the poor clinical condition, further chemotherapy and bevacizumab were stopped in favour of best supportive care.

Brain stem and spinal metastases of supratentorial glioblastoma multiforme are well known in the late course of the disease, but are rarely symptomatic. In a hospital series of 600 GBM patients, the incidence of symptomatic spinal metastases was about 2\% [12]. To date, the occurrence of an expophytic cerebellar glioblastoma in combination with a solid spinal manifestation has not yet been reported.

Fiorentino et al. described combined radiotherapy and bevacizumab in a case of supratentorial GBM with spinal manifestation. This patient died 6 months following diagnosis of metastasis and 3 months after the end of therapy [13]. No specific data have been published yet about treatments with bevacizumab of GBM with spinal manifestation.

A technical enquiry to Hoffmann-La Roche Ltd. Basel Switzerland by the corresponding author revealed that there is no data available concerning the bioavailability of bevacizumab in the cerebrospinal fluid (CSF).

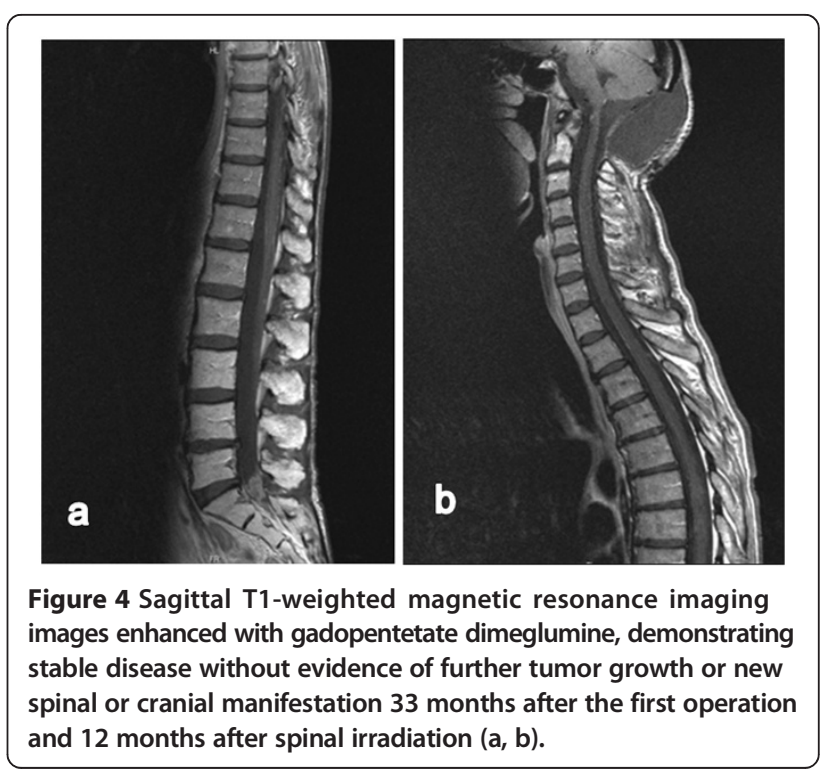


Despite the fact that the prognosis of GBM of the posterior fossa seems to be slightly better than GBM of the cerebral hemispheres, we confirmed in our case an absence of IDH -1 mutation, a negative MGMT methylation, and a meningeosis carcinomatosa as a "signum malum omnis". Altogether, these are negative predictors of a good response to chemotherapy and long overall survival.

On the other hand, an off-label-use of intravenous bevacizumab combined with temozolomide and radiotherapy arrested a further tumor growth at least for 33 months in the presented case.

\section{Conclusions}

Exophytic cerebellar glioblastoma of the posterior fossa is a rare occurrence. This is also the first report of a secondary spinal manifestation of this entity.

This report highlights the potential of a combined adjuvant therapy with bevacizumab to achieve a long-term tumor control in the case of spinal manifestation of an exophytic glioblastoma multiforme of the posterior fossa.

Due to the limited overall prognosis of the disease, spinal manifestations of glioma are rarely clinically relevant.

This is a single case study, thus we cannot postulate this therapy as standard therapy. The results of our instructive case, however, with a positive effect on both life quality and survival warrant treating future patients in the frame of a prospective clinical study.

\section{Consent}

Written informed consent was obtained from the patient for publication of this case report and accompanying images. A copy of the written consent is available for review by the Editor-in-Chief of this journal.

\section{Abbreviations}

CNS: Central nervous system; CSF: Cerebrospinal fluid; EGFR: Epidermal growth factor receptor; EMA: European Medicines Agency; EMG: Electromyographies; GEFAP: Glial fibrillary acidic protein; GBM: Glioblastoma multiforme; Gd: Gadolinium; Gd-DTPA: Gadopentetate dimeglumine; IDH-1: Isocitrate dehydrogenase 1; MEP: Motoric-evoked potentials; MGMT: O(6)-MethylguanineDNA methyltransferase; MRI: Magnetic resonance imaging; SEP: Somatosensory evoked potentials.
}

\section{Competing interests}

All authors declare that they have no competing interests.

\section{Authors' contributions}

$T L$ collected data for the case report and drafted the manuscript. CMM performed the histological examinations. AFK, CH, RIE and TW critically revised the manuscript. GHV also critically revised the manuscript and made significant revisions as native speaker. ML was involved in drafting the manuscript and gave final approval for publication. All authors read and approved the final manuscript.

\section{Acknowledgement}

This publication was funded by the German Research Foundation (DFG) and the University of Wuerzburg in the funding programme Open Access Publishing

\section{Author details}

'Department of Neurosurgery, Julius-Maximilians-University, 97080 Wuerzburg, Germany. ${ }^{2}$ Department of Neuropathology, Julius-Maximilians-University, 97080 Wuerzburg, Germany. ${ }^{3}$ Department of Neurosurgery, General Hospital, 9020

Klagenfurt, Austria.

Received: 28 November 2013 Accepted: 28 July 2014

Published: 7 August 2014

\section{References}

1. Kuroiwa T, Numaguchi $Y$, Rothman Ml, Zoarski GH: Posterior fossa glioblastoma multiforme: MR findings. AJNR Am J Neuroradiol 1995, 16:583-589.

2. Yamamoto M, Fukushima T, Sakamato S, Tsugu H: Cerebellar gliomas with exophytic growth: three case reports. Neurol Med Chir (Tokyo) 1997, 37:411-415

3. Grahovac G, Tomac D, Lambasa S: Cerebellar glioblastomas: pathophysiology, clinical presentation and management. Acta Neurochir 2009, 151:653-657.

4. Stark AM, Nabavi A, Mehdorn HM, Blomer U: Glioblastoma mulitforme-report of 267 cases treated at a single institution. Surg Neurol 2005, 63:162-169.

5. De la Fuente BP, Dalmau J, Rosenfeld M: Glioma therapy update. Neurologia 2007, 22:159-169. Span.

6. De Angelis LM: Brain tumors. N Engl J Med 2001, 344:114-123.

7. Stupp R, Mason WP, van den Bent MJ, Weller M: Radiotherapy plus concomitant and adjuvant temozolomide for glioblastoma. N Engl J Med 2005, 352:987-996.

8. Weber DC, Miller RC, Villà S, Hanssens P: Outcome and prognostic factors in cerebellar glioblastoma mulitforme in adults: a retrospective study from the rare cancer network. Int J Radiat Oncol Biol Phys 2006 66(No 1):179-186.

9. Saito T, Hama S, Kajiwara Y, Sugiyama K: Prognosis of cerebellar glioblastomas: correlation between prognosis and immunoreactivity for epidermal growth factor receptor compared with supratentorial glioblastomas. Anticancer Res 2006, 26(2B):1351-1357.

10. Utsuki S, Tanaka S, Oka M: Glioblastoma multiforme metastasis to the axis. J Neurosurg 2005, 102:540-542.

11. Cort D,BA, Nagasawa DT,MD, Isaac YMD: Leptomeningeal spinal metastases from glioblastoma multiforme: treatment and management of an uncommon manifestation of disease. A review. J Neurosurg Spine 2012, 17(5):438-448

12. Vertosick FT Jr, Selker RG: Brain stem and spinal metastases of supratentorial glioblastoma multiforme: a clinical series. Neurosurgery 1990, 27(4):516-521.

13. Fiorentino A, Caivano R, Chiumento C, Cozzolino M, Fuco V: Radiotherapy and Bevacizumab for intramedullary and leptomenigeal metastatic Glioblastoma: a case report and review of the literature. Int J Neurosci 2012, 122(11):1-9.

doi:10.1186/1756-0500-7-496

Cite this article as: Linsenmann et al: Long-term tumor control of spinal dissemination of cerebellar glioblastoma multiforme by combined adjuvant bevacizumab antibody therapy: a case report. BMC Research Notes 2014 7:496

\section{Submit your next manuscript to BioMed Central and take full advantage of:}

- Convenient online submission

- Thorough peer review

- No space constraints or color figure charges

- Immediate publication on acceptance

- Inclusion in PubMed, CAS, Scopus and Google Scholar

- Research which is freely available for redistribution 